\title{
versants
}

\section{La autorreflexión en García de Pablo García Casado}

\author{
Larissa BINDER \& Sofia SABATINI \\ Universidad de Zúrich
}

\begin{abstract}
Las estrategias autorreflexivas son muy ocurrentes en la poesía contemporánea: en el último poemario de Pablo García Casado (García Casado 20I5) conducen al yo poético al conocimiento de sí mismo y de la realidad presente mediante un viaje introspectivo que, al mismo tiempo, evoca en el nivel enunciativo tres características eminentes de la poesía: ciclicidad, tradición e innovación.

Keywords: Autorreflexión, Pablo García Casado, Poesía española del siglo xxI, García
\end{abstract}

\section{Introducción}

La poesía de Pablo García Casado destaca por su «antilirismo» o, según da a entender el autor en una entrevista, se trata de una poesía que no se centra exclusivamente en el yo; el poeta se presenta como mero «codificador de un mensaje» (1999: 47) y se considera un escritor «realista», lo que, siguiendo su propia definición, implica que se concentra más en el hecho de comunicar que el hecho de expresar1. López Guil (2019), de acuerdo con García Casado, divide la obra del poeta cordobés en dos etapas creativas: una primera de aprendizaje (integrada por los dos libros iniciales Las afueras y El mapa de América), que se centra en el dominio de la técnica con el fin de comunicar con mayor eficacia, y una segunda de conocimiento (que comprende los dos últimos poemarios Dinero y García), en la cual -como dice en el poema «Colmenas» (García Casado 2007: I66) - intenta atravesar «esa espesa gelatina que impide mirar más allá de las persianas». En una entrevista con Andrés García Cerdán afirma que el poema para él es «una luz que alumbra regiones oscuras. La luz de la emoción que también es una forma de conocimiento» (García Cerdán 20I5). Dicho de otra manera, el realismo de Pablo García Casado radica en una mirada sobre el mundo y el hombre que se pretende objetiva, o, como afirma también en la entrevista (García Cerdán 2015), aquella que en García proporciona «la conciencia de ciudadano, que mira sin ingenuidad su entorno». Pero, al mismo tiempo, el poema también ha de conmover comunicando ${ }^{2}$. Este concepto de «antilirismo realista» también es perceptible en su último libro, García (20I5). El

I «[...] puedo afirmar que soy un poeta de escritura realista, que me interesa más la comunicación que la expresividad», afirma Casado en El Ciervo (1999).

2 «Andrés García Cerdán: ¿Qué se le debe exigir a un poema? / Pablo García Casado: Qué [sic!] emocione. Que alumbre regiones oscuras. Que ahonde en la superficie de las cosas. Que sea completamente imprescindible» (García Cerdán 20I5). 
sujeto poético nos comunica y nos hace conocer, mediante una profunda introspección, la realidad sobre la sociedad que lo rodea, incluyendo también su propio entorno familiar y, por supuesto, a sí mismo. Implícitamente, además, los poemas manifiestan una profunda autor referencialidad, es decir, proponen, en su sentido figurado, una importante reflexión metaliteraria. Esto también se revela mediante otro aspecto importante en la poética de García Casado: «La disolución del sujeto poético y la asunción de las voces múltiples en lugar de una voz ha dado como resultado un complejo juego de fuerzas que ha cuestionado los límites de la propia escritura» (2005: 17). El quiebro de la omnipresencia del yo se da por varias vías: por heterónimos, por la ausencia total del yo o por «discursos en primera persona salpicados de elementos y fragmentos ajenos a él» (2005: 19).

En las páginas siguientes se analizarán dos poemas claramente autorreflexivos de García, «Todo sobre mi padre» y «Turn», representativos de cada una de las dos secciones del libro, tituladas «Yo soy mi padre» $\mathrm{y}$ «Turn».

\section{Tradición y conocimiento en «Todo sobre mi padre»}

«Todo sobre mi padre» es el tercer poema de la primera sección de García y está dividido en tres párrafos. El primero contiene seis frases y el segundo, cuatro, de modo que, visualmente, hay un cierto equilibrio en su extensión. El último fragmento, sin embargo, es más breve, de dos frases, y termina al principio del segundo renglón, como puede comprobarse a continuación:

\section{TODO SOBRE MI PADRE}

Estoy pensando en mí mismo, justo antes de nacer, sentado en la sala de espera de una maternidad. Tengo veinticinco años y ya he aprendido algunas certezas. Algunas amargas renuncias. Pero ahora eso da igual, porque ha nacido mi hijo y estoy dispuesto a jurar los Principios del Movimiento. Por este niño moreno y enfermizo. Por este amor que todavía desconozco, esta mujer que me fascina.

Estoy pensando en mí mismo, tengo veinticinco años, pronto emprenderé un largo viaje. A las playas desiertas de Salou, a los apartamentos vacíos del invierno, donde hemos sido felices, ajenos al ruido de una España mortecina. Él y yo, y esa mujer que en la foto nos abraza. Esa mujer que hoy abraza a mi hijo, también con gafas, con la misma sonrisa de mi padre.

Estoy pensando en mi hijo cuando veo a mi padre. Yo soy mi padre.

(García Casado 20I5: I4) 
En el plano del enunciado el poema trata sobre la relación entre padre e hijo, sobre recuerdos de familia, sobre la búsqueda de sí mismo y el hallazgo del yo en la figura paterna. Nuestro análisis se basa en la siguiente hipótesis: en el poema «Todo sobre mi padre» están representados dos acercamientos metaliterarios enlazados. Por un lado, se verifica la afirmación de García Casado respecto a la influencia que ejerce la tradición en la escritura de cada poeta y, por otro lado, se concibe la escritura poética como acto de conocimiento.

Al inicio de los dos primeros párrafos se repite de forma anafórica la misma oración «Estoy pensando en mí mismo»; sin embargo, en el último párrafo se produce una significativa variación en el objeto del pensamiento, que ya no es «mí mismo» sino «mi hijo». Otro paralelismo entre los dos párrafos iniciales es la repetición de las palabras «tengo veinticinco años», que establecen el tiempo interno del poema y la edad del yo poético. Un indicio temporal que esta vez nos permite situar el poema en una época específica es la disposición del yo poético de la primera estrofa a «jurar los Principios del Movimiento», a saber, una de las siete Leyes Fundamentales del régimen de Franco que establecía, como su nombre indica, los principios en los cuales estaban basados los ideales de patria, familia y religión, junto con el máximo respeto al régimen franquista instaurado en aquel entonces. El tiempo interior es, por tanto, posterior a I958, fecha en la que fue promulgada dicha ley.

La voz poética de los tres párrafos relata empleando verbos en primera persona: «estoy pensando», «tengo veinticinco años», «he aprendido», «estoy dispuesto», «desconozco», «estoy pensando», «tengo veinticinco años», «emprenderé», «estoy pensando», «veo», «soy». No obstante este paralelismo verbal, hay indicios de que se trata de voces poéticas múltiples, siguiendo lo que García Casado llama «la disolución del sujeto poético y la asunción de las voces múltiples en lugar de una voz» (2005: I7). Como se verá en lo que sigue, el yo del segundo párrafo (yoz) es el hijo que aparece en el primero y entre los dos párrafos hay una diferencia temporal de veinticinco años. Un indicio es el viaje que el yoz planea. Por un lado, es un acontecimiento vuelto hacia el futuro, según denotan el adverbio «pronto» y la forma verbal «emprenderé». Por otro lado, no se trata solamente de un viaje físico a otro lugar, sino también de un viaje mental a los recuerdos de la infancia, de vacaciones en familia, recuerdos «felices» y «ajenos a la España mortecina» que claramente apunta a una España bajo el régimen fascista y conecta los recuerdos insinuados en la segunda parte con la primera parte, cuyo tiempo interno se desenvuelve durante el fascismo. Los sujetos del verbo "hemos sido felices» son, como se expone en la frase siguiente, el yo2 poético y sus padres «Él y yo, y esa mujer que nos abraza». El demostrativo a distancia «esa» remite deícticamente a la misma mujer que en el primer párrafo se modifica con el 
demostrativo de mayor cercanía «esta». Este cambio de deíctico de «esta»a «esa» se debe a que la relación que los yos mantienen con la mujer no es la misma: para el primer yo (yoI), la mujer es la esposa, o, por lo menos, la pareja amorosa por la cual el yor siente fascinación ("esta mujer que me fascina»). Para el yo2, el personaje «mujer» es la madre. Mientras que «en la foto» la mujer abraza al padre y a su hijo, en el tiempo considerado 'actualidad' en la segunda estrofa, abraza a su nieto, o sea, al hijo del yo2 poético: «Esa mujer que hoy abraza a mi hijo».

En el último párrafo («Estoy pensando en mi hijo cuando veo a mi padre»), por primera vez los pensamientos se centran en un ser que ya no es el yo poético, ni el yor ni el yoz, es decir, no dice «estoy pensando en mí mismo» como en las estrofas precedentes, sino en otra persona que es el hijo del yo2 o el nieto del yor. También acontece que, por primera vez, los tres personajes principales, padre, hijo y nieto, están presentes en una sola frase y están conectados por el pensamiento y la mirada del yo2, formando así una unión triádica que evoca la santísima trinidad. La última frase «Yo soy mi padre», además de establecer otro lazo con la Biblia -pues, en el Evangelio de San Juan, Jesús afirma: «Yo y el Padre uno somos» (Biblia I960)-, y es una importante clave de lectura, según veremos a continuación.

Una vez que el lector relee el poema, entiende que el yor es el padre del yo2, y que «mí mismo» no es exclusivo de una única persona. Eso lo prueba también la oración apositiva «justo antes de nacer», que debería aportar información sobre cuándo piensa el yor en sí mismo en el primer párrafo, pero resulta ambigua. Por un lado, el infinitivo «nacer» debería gramaticalmente depender del mismo sujeto que el verbo «estoy pensando». Pero, después de la primera lectura, sabemos, que quien está por nacer es el hijo de quien habla (el yo del segundo y tercer párrafos), y no él mismo, el yor. Aquí hay una transitividad que une las acciones de padre e hijo sin que percibamos quién es verdaderamente el sujeto de «justo antes de nacer».

En función de los datos anteriores, el texto puede segmentarse discursivamente en dos macrosecuencias: la primera de ellas, que llamaremos A, comprende el título, sobre el cual se hablará a continuación, mientras que B abarcaría los tres párrafos. En Bi, primero y segundo párrafo, los dos yos son la voz de los padres jóvenes que cuentan una nueva etapa de su vida, debido al nacimiento de un hijo. En BI. I (primer párrafo) el yor es padre del quien dirá yo2 en BI.2 (segundo párrafo) y, por tanto, abuelo de a quien el yo2 llama «mi hijo». Solo en la parte B2, a través de los acontecimientos de BI, se manifiesta un momento de identificación total por parte del yo2, cuando se da cuenta de que la contemplación del padre («cuando veo a mi padre») lo hace pensar en el propio hijo («estoy pensando en mi hijo»), y en su misma existencia como padre («yo soy mi padre»), generando empatía entre todos los personajes. En otra entrevista de 20I5, García Casado dice: «[...] la figura del 
padre es también metáfora de relaciones de poder, porque revela también muchos miedos contemporáneos. Y porque pone de manifiesto preguntas sobre la identidad, puesto que uno puede ser padre e hijo al mismo tiempo. Porque te pareces a tu padre, tu hijo se parece a ti, tu hijo a tu padre... hablamos de sujetos plurales, confundidos, diluidos entre sí» (De la Fuente 20I5). La afirmación «tu hijo [se parece] a tu padre», se evoca con claridad en la última frase del segundo párrafo cuando el yoz habla de su hijo y dice que tiene «la misma sonrisa de mi padre». Identificarse, entonces, es conocer a los otros y a sí mismo, estableciendo un lazo empático que conecta todas las generaciones.

Volviendo al título, que forma la macrosecuencia A, «Todo sobre mi padre» alude indudablemente a la película casi homónima del cineasta Pedro Almodóvar «Todo sobre mi madre» (1999)3 ${ }^{3}$, cuyo movimiento de identidad es parecido al de nuestro poema: en ella se presenta a un hijo, Esteban, a la búsqueda de su padre, también Esteban o Lola, el nombre femenino, dado que se trata de una persona transexual. El nombre del hijo, igual al del padre, establece un lazo no solo genético sino también antroponímico con ayuda de la madre, que lo bautiza con el nombre del padre. El hijo muere al inicio de la película y lo que se cuenta, es cómo la madre y protagonista de la obra, Manuela continúa la misión del hijo de encontrar al padre, para informarle de la existencia y al mismo tiempo de la muerte del hijo común. A lo largo de esa búsqueda, Manuela acepta una propuesta de trabajo como asistente personal de una actriz teatral famosa y preferida del hijo fallecido, Huma Rojo. Cuando una noche la colega de Huma, Nina, está de baja por una sobredosis y no puede actuar, Manuela se ofrece inocentemente a reemplazarla para no cancelar la presentación, dado que conoce de memoria el papel. Con su actuación, Manuela se gana la admiración por parte del director y del público. Ese acontecimiento remite a su vez, al origen del nombre y de la idea de la pieza cinematográfica, que es una película estadounidense del director Joseph L. Mankiewicz y se llama «All about Eve» (I950) ${ }^{4}$, en que la protagonista, Eve, asciende a ser una célebre actriz, a través de la intrigante táctica de reemplazar a la estrella de teatro, Margo, de la cual había sido la secretaria. El vínculo entre las dos películas resulta evidente: al principio de «Todo sobre mi madre» Manuela y Esteban-hijo ven juntos «All about Eve» en la televisión y, a lo largo de la historia, se establece un paralelismo en el comportamiento entre los personajes Manuela e Eve que determina el título

3 Almodóvar, Pedro \& Almodóvar, Augustín (productores) \& Almodóvar, Pedro (director) (1999), Todo sobre mi madre, España, El Deseo, con Cecilia Roth, Marisa Paredes, Candela Peña, Antonia San Juan, Penélope Cruz.

4 Zanuck, Darryl F. (productor) \& Mankiewicz, Joseph L. (1950), All about Eve, Estados Unidos, 2oth Century Fox, con Bette Davis, Anne Baxter, Gary Merrill, George Sanders, Hugh Marlowe, Celeste Holm, Thelma Ritter, Marilyn Monroe. 
igual al de «All about Eve». Volviendo al poema, el título «Todo sobre mi padre» remite al personaje principal, como lo es Eve y como lo es la madre, también presentes en el título, que en el poema es la figura paterna, el yor, si bien el yoz es el referente del adjetivo posesivo «mi». Además, en la película de Almodóvar, el hijo, antes de morir, le revela a su madre que desea escribir un cuento sobre ella. El poema que sigue al título resulta ser justamente lo que había planeado Esteban en «All about Eve», esto es, un homenaje a quien crió al yo poético.

Pasemos ahora a la macrosecuencia B, donde encontramos un embrague textual, ya que el texto está representando icónicamente lo que dice, produciéndose una identidad entre lo dicho y lo representado, entre el número y la extensión de los párrafos y los 3 sujetos de los que se habla en cada uno de esos párrafos: un padre a los 25 (yor en BI.I), un hijo a los 25 (yoz en Bı.2) y un nieto muy pequeño, recién nacido, en B2. Los tres párrafos forman el poema, de la misma manera que los tres seres humanos forman una continuidad en el tiempo y en el espacio. Además hay un orden genealógico en el texto: el párrafo que habla del padre yor es el primero, el que se dedica al hijo yoz es el segundo y aquel en el que se transforma la anáfora inicial («Estoy pensando en mí mismo») por algo parecido pero diferente («Estoy pensando en mi hijo»), y en donde el nieto adquiere protagonismo, es el tercer párrafo, el más breve en extensión, ya que el nieto es el más joven de los personajes y no tiene aún los 25 años del yor y el yoz. De ese modo, aunque el nieto, representado por el párrafo más breve, sea aún pequeño, la repetición anafórica de buena parte de la frase inicial pone de manifiesto no solo lo diferente (dos palabras y por tanto cuantitativamente menor), sino sobre todo lo común (3 palabras: «estoy pensando en»), que es cuantitativamente mayor y lo conecta a los dos personajes anteriores.

Teniendo en cuenta lo afirmado anteriormente respecto al título y la representación icónica del enunciado en los tres párrafos, parece lícito realizar una lectura autorreferencial del enunciado, en tanto en cuanto el poema parece estar hablando también de sí mismo. El padre es alguien (o algo) que ha existido antes del propio nacimiento. Alguien que se encuentra en nuestros pensamientos, en nuestros recuerdos y en nuestro ADN; alguien que nos ha nutrido, tanto en el sentido literal como en el metafórico. Es alguien (o algo) que también tuvo un padre, un origen, unos recuerdos de su propio padre y así sucesivamente en la cadena generacional hacia el pasado. García Casado afirma en una entrevista que cada poeta se nutre de la tradición: «La originalidad no existe. Todos bebemos de la tradición, ya sea por adhesión o por rechazo. Lo que ocurre es que el concepto de tradición se está ensanchando cada vez más. Tradición es Góngora, Velázquez o Mozart, pero también lo son Sex Pistols, Baccara, Tinto Bras o los anuncios de detergentes» (I999: 47). El hecho de que la tradición resulte omnipresente en las artes y en este 
poema, se muestra claramente desde el comienzo con el título inspirado en otra pieza artística, la película de Almodóvar, donde encontramos la misma dinámica de interiorizar al padre y la tradición que en el poema. Algo que sucede igualmente en el intertexto, pues también «Todo sobre mi madre» se basa en otra pieza, esto es, en «All about Eve». El padre de Esteban, el adolescente que muere al principio, es también padre de otro chico que recibe de nuevo el nombre Esteban. Este hecho muestra que toda creación nueva -como lo son el recién nacido Esteban y, mutatis mutandi, el nieto de nuestro poema- deriva del padre, esto es, de la tradición. Respecto al relato, esta misma dinámica se muestra mediante la sustitución de las famosas actrices Margo y Nina por los personajes Eve y Manuela, sendas protagonistas de ambas películas: las dos interiorizan los papeles de sus ídolos, interpretándolos de manera formidable, aunque no haya ninguna relación sanguínea entre ellas. Si las célebres actrices representan, en un nivel meta-artístico, la tradición, eso muestra que no es necesario que la fuente de inspiración tradicional sea del mismo género que la nueva creación. Al igual que Manuela puede reemplazar a Nina sin que haya un lazo directo entre ellas, una película puede ser reinterpretada en un poema u otra expresión artística. La tradición, entonces, siempre influye en las nuevas creaciones (poéticas) y un artista no puede desprenderse de ella, como el hijo nunca se podrá desconectarse genéticamente del padre. Ahora bien, si el padre, retratado en el primer párrafo y encarnado textualmente por este fragmento, podría estar representando la tradición, entonces el hijo, figurativizado en y por el segundo párrafo, podría estar encarnando la nueva poesía, la creación poética en curso. Hablamos de poesía y no de poemas, porque el segundo yo poético, el yoz que retoma la palabra en BI.2 es representado como padre e hijo a la vez. En una entrevista hablando de Luís García Montero, García Casado dice lo siguiente: «Yo creo que ha pasado el momento de "matar a los padres", entre otras cosas porque esa etapa se corresponde con un aislacionismo cultural en la que tus referentes son tus padres literarios, de los que o bebes o rechazas. Un poeta, en el siglo xxI, bebe de muchas fuentes diversas, de muchas tradiciones literarias y de muchas épocas, y también de otras disciplinas culturales y de ese enorme magma informativo en el que estamos inmersos» (Acebes 20I4). De la misma manera que todo hijo es también su padre, esto se perpetuará siempre, también en la poesía futura, la aún no escrita y figurativizada por la brevedad del último párrafo. La afirmación «Yo soy mi padre», tendría, pues, una lectura metapoética según la cual se admite formar parte de la tradición. Pero esa inclusión, solo acontece a partir de un hecho dado que es el nacimiento del hijo, o bien, en términos literarios, a partir de una nueva escritura poética. 


\section{Linealidad y ciclicidad en «Turn»}

«Turn» es el penúltimo poema de la sección homónima y al igual que el resto de las composiciones del libro, está escrito en prosa:

I

Éramos felices, teníamos trabajo. El pasado era una suma de derrotas necesarias, un lugar al que nunca regresaríamos. Había entre nosotros la certeza de un perpetuo crecimiento. Los vientres se llenaron de vida, y todo el mundo era padre y madre, con esa alegría que sólo tuvieron los primeros pobladores de la tierra. Nadie sentía el miedo de sus antepasados. Los viejos contemplaban orgullosos el mundo que entregaban a sus hijos. Y éstos apuraban hasta la última gota. Éramos felices, teníamos trabajo.

II

La fiesta se acabó. La noche empezó a caer, lentamente, sobre las estadísticas: Dejaron de llegar manjares del trópico, se llenaron las tiendas de productos de plástico. La gente se quedó en casa a esperar que cesara la lluvia. Y fueron apagando los braseros. Y empezaron a comer más pan y a tragarlo más despacio. Y volvieron los remiendos y las pequeñas reparaciones. Y los coches se hicieron viejos, ruidosos, moribundos. Y volvimos a ser de nuevo este país que espera un milagro. Y volvimos a vestir a nuestros hijos de uniforme.

\section{III}

Hay un tiempo para todo, bajo el cielo, un tiempo para cada cosa. Y el tiempo es ahora, y es aquí. He de encontrar un relato, una certeza. No quiero una rendición sin condiciones. Quiero decirle a mis hijos: aquí tenéis la mañana, es toda vuestra, sin duda os pertenece.

(2015: 45-46)

Compuesto por tres párrafos de diferente extensión, numerados con cifras romanas, en el plano del enunciado, el poema describe tres momentos distintos. I versa sobre el pasado despreocupado, el momento antes de la crisis económica, según se deduce de la frase repetida al inicio y al final de la primera estrofa («Éramos felices, teníamos trabajo»), que remite obviamente a un tiempo en el que nadie había de preocuparse por el futuro económico, pues «tenían trabajo». En II está descrito el cambio como un proceso dinámico e imparable, y en III -que se inicia con la expresión presentativa e impersonal «hay» que implica un salto temporal hacia el presente-, el yo poético expresa el deseo de procurarles una «mañana», o sea un futuro, a sus hijos. El poema ofrece, por una parte, una perspectiva global, la evo- 
lución de la humanidad desde «los primeros pobladores de la tierra» hasta el actual presente y, por otra parte, denuncia la evolución de la España sociopolítica, como afirma Gonzalo Gragera (2017) en un artículo sobre el poemario García: «en el segundo tramo del conjunto nos damos de bruces con el retrato de la España sociopolítica de los últimos años -corrupción, paro, frases hechas y clichés de los políticos en las ruedas de prensa-y con algún que otro manifiesto que esconde una sutil pero contundente carga crítica».

Como se espera mostrar a continuación, «Turn» pone de relieve la oposición entre la efímera linealidad de la vida humana, en la que todo tiene su tiempo irrepetible, señalando tres momentos distintos en la historia sociopolítica y económica de España, y la circularidad propia de la naturaleza cíclica del arte, susceptible de ser actualizado una y otra vez.

En lo concerniente al título del poema, la expresión «turn» es muy ambigua y tiene al menos tres significados. No nos sorprende, sin embargo, que sea éste el poema que da título a la segunda sección del libro ya que también puede leerse como forma de imperativo, una suerte de convocatoria al cambio. Por otra parte, también podría interpretarse como infinitivo o sustantivo remitiendo así al cambio descrito sobre todo en II, que acabamos de mencionar. Siguiendo nuestra hipótesis, el significado de la palabra «turn» también puede interpretarse como un proceso cíclico de una vuelta o rotación, lo que remitiría entonces al carácter cíclico del arte.

Atendiendo a los tiempos verbales, al grado de dinamismo indicado por la semántica de los verbos, a las formas personales y las figuras retóricas empleadas, podemos segmentar discursivamente el texto en dos secciones: l y II, en pasado, constituirían la primera macrosecuencia A, a su vez divisible en AI (I) y A2 (II), según se tratará de demostrar en lo que sigue. III, en presente, integraría la segunda macrosecuencia, que llamaremos B.

En A, las formas verbales remiten a acciones o estados pasados con una diferencia de aspecto temporal: en Ar abunda el imperfecto, el aspecto de 'acción o estado continuos', aspecto verbal que implicaría cierta ciclicidad, $\mathrm{y}$, en A2, aparecen las formas del perfecto indefinido, con su aspecto de 'acción concluida'. Además, la frontera entre Ar y A2 está marcada por un embrague textual al inicio de II, «La fiesta se acabó», que evoca la época feliz descrita en Ai y cuyo fin se representa tipográficamente a través del espacio blanco que media entre la primera y segunda estrofas. Esta frase, además, desmiente la constatación afirmada en Ai de que «el pasado era una suma de derrotas necesarias, un lugar al que nunca regresaríamos»: el cambio o bien los cambios que se describen en A2 son la prueba de que la crisis puede volver. Sin embargo, el cambio más abrupto se encuentra entre II (A2) y III (B) con la actualización y vuelta al presente, al tiempo de la narración.

En la parte Ai se describe un pasado feliz que se caracteriza por cierta estabilidad y nos hallamos frente a un proceso cíclico dentro de la linea- 
lidad. Este hecho se refuerza por la repetición de la frase «Éramos felices, teníamos trabajo» al inicio y al final de la estrofa. Cierto, este proceso es de una ciclicidad ficticia puesto que se pierde en A2. Sin embargo, la capacidad de referirse positivamente a aquella época es el fundamento de esta felicidad. Si comparamos el plano del enunciado y el plano de la enunciación en AI, constatamos, entonces, una correspondencia bastante notable: coincide el modo de decir (la repetición de la primera frase) con lo que se dice. La conclusión a la que se puede llegar analizando la primera estrofa consiste en una fuerte valoración del momento anterior a la crisis por su estabilidad cíclica, ciclicidad comparable al arte con sus diversas manifestaciones. En lo tocante al dinamismo implicado en la semántica de los verbos, Ar se caracteriza por la abundancia de verbos que indican un estado más bien estático, como los verbos «ser», «tener» 0 «contemplar».

Pasando de Ar a A2, el cambio abrupto del imperfecto al indefinido repentinamente presta dinamismo a un relato, en el que, además, los verbos son de acción (por ejemplo, «acabar», «empezar», «dejar», «volver» o «hacer»). Dicho dinamismo pone de manifiesto la velocidad del cambio, la pérdida de la felicidad, reforzada sintácticamente con la abundancia de yuxtaposiciones y de frases simples. La construcción anafórica de la conjunción «y» con un verbo en indefinido contribuye a una lectura rítmica, de modo que los acontecimientos narrados parecen acelerarse de forma progresiva e imparable hasta llegar al estado presente (B), donde se produce una vuelta a un estado estático, un presente sin dinamismo (evocado ya en A2 con la siguiente oración: «volvimos a ser de nuevo este país que espera un milagro»). La mayoritaria sintaxis paratáctica evoca, sin embargo, un aire de monotonía, de repetición debido también a la reiterada construcción anafórica con $y+$ verbo de tercera persona del plural. La crítica sociopolítica se ve sobre todo en el plano del contenido, ya que en A2 ocurren muchos términos que implícitamente remiten al campo semántico de la economía («las estadísticas», «las tiendas», «los coches»...). La anáfora («Y volvimos [...]. Y volvimos [...]») sirve de conclusión de la estrofa. Dicho de otra manera, el empleo anafórico del verbo «volver» refuerza aún el carácter conclusivo que denota su semántica, a saber «poner o constituir nuevamente a alguien o algo en el estado que antes tenía» según la definición del DRAE, que señala, por consiguiente, que el proceso de cambio ha terminado. En lo que a las formas personales respecta, constatamos que en la primera estrofa abunda la primera persona de plural, un «nosotros» en el que el yo, claro está, se incluye. Este «nosotros» desaparece al inicio de A2, donde se emplean mayoritariamente formas de la tercera persona, descritas como «formes de la non-personne» por el lingüista Benveniste (I966), por ser formas personales con referentes no presentes en el momento de la conversación, y vuelve a aparecer en las 
últimas dos frases: «Y volvimos a ser de nuevo este país que espera un milagro. Y volvimos a vestir a nuestros hijos de uniforme».

En B, sin embargo, la primera persona del plural desaparece y cede el paso a un yo que se distancia del «nosotros», o sea, de la sociedad que se rinde «sin condiciones». Lo que desea este yo es ofrecerles un futuro próspero a sus hijos: «aquí tenéis la mañana [...]». Otra característica de la parte B es una fuerte polifonía textual. Fijémonos en la siguiente oración: «Hay un tiempo para todo, bajo el cielo, un tiempo para cada cosa». Esta oración retoma la idea de un texto bíblico (Ecclesiastes III: I-8) y de la letra de una canción de Pete Seeger («Turn! Turn! Turn!», I965) que a su vez se menciona al inicio de la segunda sección de diez poemas. Al comparar los dos textos a los que la tercera estrofa remite, la canción de Seeger (I965) se distingue del texto bíblico por tener un toque de esperanza al final: «A time for peace, I swear, it's not too late». Este toque de esperanza también se halla en las palabras del poema de García Casado, o al menos una determinación para el cambio, implicada en las expresiones de deseo «No quiero» o «quiero». La etimología del verbo «querer» denota el concepto de buscar o procurar también y remite, por lo tanto, a la firmeza de cambio. Lo que distingue, entonces, Ai y B es la valoración que les da el sujeto poético: mientras que A s se nos presenta como un tiempo feliz en el cual «había entre nosotros la certeza de un perpetuo crecimiento», mientras que el presente parece ser su gemelo maligno que, a pesar de mostrar cierta ciclicidad, se caracteriza al mismo tiempo por una falta de dinamismo, vista desde una perspectiva más negativa. Sin embargo, no diríamos que sea una perspectiva pesimista puesto que se nota un toque de esperanza en $B$, parecido al final de la canción de Seeger, cuando el sujeto poético anuncia que «[ha] de encontrar un relato, una certeza» y que no «[quiere] una rendición sin condiciones», lo que implica que aún estamos a tiempo, o sea, al menos, que el sujeto poético aún no ha perdido la esperanza.

Acabamos de ver una suerte de lamentación del pasado al que no se puede regresar en la parte A y una crítica de la situación actual en la que se «espera un milagro» en la parte $B$, pues el verbo esperar cuya semántica, según el DRAE, denota «no comenzar a actuar hasta que suceda algo». Esto ya se ve en Ai cuando el yo poético dice, por ejemplo, «esa alegría que sólo tuvieron los primeros pobladores de la tierra». El adverbio «sólo» implica que «esa alegría» fue exclusivamente propia de «los primeros pobladores»y excluye a todos los demás seres humanos. Nuestro análisis también ha proporcionado una perspectiva sobre la linealidad de la vida humana incluyendo la idea de la canción de Seeger: todo tiene su tiempo, y cada tiempo tiene su propio relato. Ello nos permite ver que la felicidad no depende únicamente de los hechos históricos y económicos, sino también del modo de relatar: si logramos a crear un relato esperanzado, creamos nuestra propia felicidad. 
En este sentido, la poesía, o sea la creación artística, sería una suerte de remedio contra la realidad decepcionante en la que no solo el poeta, es decir el artista, es capaz refugiarse. En otras palabras, la creación poética, la fuerza de las palabras y de las manifestaciones artísticas, no solo es capaz de procurarnos una mañana tan feliz como el pasado de Ar sino que son necesarias para revertir el signo negativo de los tiempos y crear un futuro positivo.

\section{Conclusión}

En los dos poemas que hemos analizado, la voz poética relata una profunda y detallada reflexión familiar y social, recordando el pasado feliz («hemos sido felices» en «Todo sobre mi padre» y «éramos felices» en «Turn»), que lleva al conocimiento de sí mismo y de la realidad presente que lo rodea. Ese viaje introspectivo y el entendimiento alcanzado se dejan trasladar, mediante nuestro análisis, a un nivel metaliterario, un nivel de profunda autorreferencialidad, donde se inscriben algunas reflexiones del autor acerca del papel que cumplen en la poesía ciclicidad, tradición e innovación.

\section{Bibliografía}

Acebes, David (20I4), «Entrevista con el hombre [hu]eco: Pablo García Casado», en línea bajo: http://www.elcotidiano.es/entrevista-con-el-hombre-hueco-pablo-garcia-casado/ [23 de diciembre 20I8]

Almodóvar, Pedro \& Almodóvar, Augustín (productores) \& Almodóvar, Pedro (director) (I999), Todo sobre mi madre, España, El Deseo.

Benveniste, Émile (I966), Problèmes de linguistique générale, Vol. I, París, Gallimard.

Biblia (I960), Sociedades Bíblicas en América Latina, Versión Reina-Valera, en línea bajo: https://www.biblegateway.com/passage/?search=Juan+IO\%3A30-33\&version=RVC [27 de diciembre 20I8]

Blesa, Tùa (20I5), «García. Pablo García Casado», El Cultural, en línea bajo: http://www.elcultural.com/revista/letras/Garcia/36426.

De la Fuente, Manuel (20I5), «Pablo García Casado: "La poesía no sólo debe ser lamento estéril, debe ser ciudadana”, en línea bajo: https://www.abc. es/cultura/libros/20150425/abci-poetas-veinticinco-abril-20I504240803. html [28 de noviembre 20I8]

García Casado, Pablo (1999), «No concibo la poesía como un arte de venusinos o iluminados», El Ciervo 48 (576), pp. 47-50.

García Casado, Pablo (2005), «Responsabilidad y disolución», Oihenari 20, pp. I7-2I.

García Casado, Pablo (20I5), García, Madrid, Visor. 
García Cerdán, Andrés (20I5), «La ciudadanía poética de Pablo García Casado», La Galla Ciencia, en línea bajo:

http://www.lagallaciencia.com/20I5/I2/la-ciudadania-poetica-de-pablo-garcia.html [26 de noviembre 20I8]

Gragera, Gonzalo (2017), «Pablo "García” Casado: Renovación y originalidad», Ocultalit, en línea bajo:

https://www.ocultalit.com/poesia/pablo-garcia-casado-garcia/ [26 de noviembre 20I8]

Lama, Juan Gabriel (2006), «Pablo García Casado», Maquinista de la Generación $\mathrm{I} 2$, pp. I26-I29.

López Guil, Itzíar (20I9), «Las afueras del poema en la obra inicial de Pablo García Casado», Actas del Congreso Internacional El título del poema (Mayo de 20I8, Univ. de Zúrich), Berna, Peter Lang, en prensa.

López Luaces, Marta (2007), «Poesía española contemporánea: crítica y poesía», Hofstra hispanic review 2(4), pp. 9-24.

López-Vega, Martín (2013), «Todo son afueras: Pablo García Casado», El Cultural, en línea bajo:

https://www.elcultural.com/blogs/rima-interna/2013/o7/todo-son-afueras-pablo-garcia-casado/ [23 de diciembre 20I8]

Prieto de Paula, Ángel L. y Langa Pizarro, Mar (2007), Manual de literatura española actual (de la transición al tercer milenio), Madrid, Castalia.

Ruiz Villagordo, Guillermo (2004), «El despojamiento humano», Lateral: Revista de Cultura II(II7), p. 20.

Zanuck, Darryl F. (productor) y Mankiewicz, Joseph L. (director) (I950), All about Eve, Estados Unidos: $20^{\text {th }}$ Century Fox. 